\title{
Insights from chironomid oviposition is useful to visual pest control
}

\author{
Amit Lerner', Carynelisa Haspel ${ }^{2}$, Nir Sapir, Nikolay Meltser', Meir Broza ${ }^{4}$ and Nadar Shashar'
}

\begin{abstract}
Lerner A, Haspel C, Sapir, N, Meltser N, Broza M and Shashar N. 2012. Insights from chironomid oviposition is useful to visual pest control. Fauna norvegica 31: 65-70.
\end{abstract}

Efficient visual pest control is still in its infant stages. Although being non-invasive, environmentally friendly and potentially cost effective, we still have not cracked the way to efficiently use it against epidemic vector-carrying and crop-infesting insects. Chironomid ovipostion can provide insights to key factors in visual pest control by investigating the behavior under confined and unconfined conditions. Under confined condition, due to limited amount of oviposition sites and increase of egg density in highly preferred locations, females oviposit in less suitable sites. Intensity and polarization of light reflected from the oviposition sites were found to be important as guiding cues for the females. However, in the open outdoor where oviposition sites are unlimited, oviposition followed the polarization signal only, and the intensity was ignored as a cue. By applying this approach to reduce chironomid population in a natural reservoir we show that it may be used to divert ovipositing females to egg traps reflecting highly and horizontally polarized light. The potential of using color, intensity and polarization as a basis for building oviposition traps and applying it to pest control in the future, is discussed.

doi: 10.5324/fn.v31i0.1404. Received: 2011-11-02. Accepted: 2012-06-24.

Published on paper and online: 2012-10-17.

Keywords: Chironomus transvaalensis, Polarization Vision, Insect control

1. Department of Life Sciences, Eilat Campus, Ben-Gurion University, Hatmarim St, 88000 Eilat, Israel

2. The Fredy and Nadine Herrmann Institute of Earth Sciences, The Hebrew University of Jerusalem, Jerusalem, 91904, Israel

3. Department of Integrative Biology, University of California, Berkeley, 3060 Valley Life Sciences Building, Berkeley, CA 94720-3140, USA

4. Department of Biology, Faculty of Science and Science Education, University of Haifa at Oranim, 36006 Tiv'on, Israel

Corresponding author: Amit Lerner

E-mail: amit.lerner35@gmail.com

\section{INTRODUCTION}

Insects can be major pests to human well-being and in agriculture. To date, chemical and biological control are the most commonly used methods to overcome insect infestation. Chemical control, although highly effective, is now becoming less and less accepted as we start to realize its negative effect on the environment as well as on our own health. Throughout the world, people are seeking for alternative control solutions that will allow decreasing or replacing the use of chemicals in agriculture. Biological control includes introducing a new organism to the ecosystem which feeds on the infesting pest, infects or otherwise limits or competes with it over resources. Although this method is widely in use, it still has its down sides. The efficiency of biological control depends on the density of the pest, it risks the introduction of invasive species, and it might be ineffective due to the competitive ability of the introduced species. Both approaches are expensive and in many cases provide only a partial solution.

Chironomids are distributed worldwide, occupying a range of fresh water habitats (Armitage et al. 1995). They often become nuisance to humans in several ways. Economically, they may be a problem as they stick to paint and to food processed in the industry and damage the final product, or by decreasing tourism in hotels nearby infected places. Health-wise, they may be inhaled, and become a source for respiratory diseases (see review in Armitage et al. 1995). Chironomids has been found 
to host Vibrio cholerae (Broza \& Halpern, 2001; Halpern et al. 2004; Broza et al. 2008), an important pathogen of the Cholera disease. Although the epidemic strain of the pathogen has not been found on any chironomid species yet (the lethal nonepidemic strains were found), it is likely that it will be in the near future (Malka Halpern, pers. comm.). Since $V$. cholerae may spread by winds between continents (Paz \& Broza, 2007) it is epidemically important to understand the biology of the chironomids and their oviposition behavior to be able to apply it to pest control. Therefore, we aim in this review to summarize our recent findings regarding the visually-guided oviposition behavior of Chironomus transvaalensis Kieffer, 1923 (Diptera: Chironomidae; non-biting midge), and to provide insights from these findings to visual control of insect pests.

\section{Visual cues guide chironomids to oviposition sites}

Chironomus larvae have the potential to infest water supply systems (Broza et al. 1998). Since chironomids of the species Chironomus transvaalensis were found to host $V$. cholerae, the motivation to study this species increased. Females of $C$. transvaalensis, mate during dusk at midair and descend to oviposit soon after. Most of the females lay one egg batch (EB) early in the night containing roughly 700 eggs enclosed in a gelatinous sack. The female attach the EB to the air-substratewater interface such as pond edges. Some of the females (ca. $30 \%$ ) wait until the next dawn to oviposit. The females die soon after they oviposit, and usually live not more than 24 hours.

An investigation of $C$. transvaalensis oviposition preference revealed that unlike their biting relatives the mosquitoes (Culicidae), the chironomid female choose its oviposition site not by odor but visually (Meltser et al. 2008; Bernáth et al. 2008). Visual cues can include the three characteristics of light, intensity, color, and polarization. Any one of them or a combination of more than one could serve as a guiding cue for the females. Chironomids are mainly sensitive to the UV and the green parts of the spectrum (Kokkinn \& Williams, 1989). In addition, the ability to sense the light's polarization is well known among arthropods. Their photoreceptors in the retina include a structural parallel organization of the microvilli inhabiting the retinal molecules that absorb the light, which allows for the analysis of incoming linearly polarized radiation (reviewed in Horváth \& Varjú 2004). Such an arrangement was found in the retina of $C$. transvaalensis where the microvilli in their photoreceptors were aligned in three orientations with a $45^{\circ}$ difference to each other (Lerner et al. 2008). Moreover, chironomids have been shown to be attracted to polarization reflecting traps (Danthanarayana \& Dashper 1986; Schwind 1991; Horváth et al., 2011). In Lerner et al. (2008), the studied water reservoir (waste stabilization pond) reflected gray light at dusk which mainly depended on the water's content and was wavelength independent. Therefore the water color was excluded as a guiding cue. Next, the two other characteristics, intensity and linear polarization, were investigated to determine which one of them could be the dominant guiding cue. Lerner et al. (2008) conducted two experiments to investigate this. One was a controlled experiment performed inside a tent where every evening at dusk, chironomid females were lured to enter the tent that contained four egg traps as oviposition sites reflecting a combination of high and low level of intensity, and polarized and unpolarized light. The spectra of the intensity and the polarization reflected from the traps were measured using a multispectral polarimeter composed of spectrophotometer attached to an optic fiber (for details see Lerner et al., 2008). The EB laid in the traps (Figure 1) were counted in the next morning, and the traps were cleaned from eggs for the next evening. The egg batches (EB) distributed on average as 10\%, 25\%, 25\%, and $40 \%$ in the unpolarized low intensity, unpolarized high intensity, polarized low intensity, and polarized high intensity reflecting traps. Therefore, most of the EB $(65 \%)$ were laid in the polarized traps. However, the unpolarized traps were not completely ignored $35 \%$ of the EBs laid in both unpolarized traps). Thus, both polarization and intensity attracted the females to oviposit in the traps. The second experiment was conducted outdoors on the pond's edge. Four black plastic tubs served as the oviposition traps and were filled with tap and pond water to reflect two levels of intensity and $0.0,0.2,0.35$ and 0.38 partial polarizations. Here, EB counts followed the level of polarization regardless of the level of intensity. In this experiment, unpolarized and high intensity reflecting egg traps were mostly ignored by the ovipositing females (only few EBs were found in these traps and their counts were statistically not different from zero; see Lerner et al., 2008, for raw EB counts). Polarization was found to be positively correlated with the amount of suspended organic carbon in the water on which the larvae feed (Lerner et al. 2008). As such, polarization can provide the female an indication regarding the amount of food available to its offspring. The relative contribution of the suspended carbon and the carbon in the bottom of the pond on the reflected polarization deserves further investigation. The polarization reflected from the pond surface, unlike the intensity, was found to be stable and constant throughout sunset, hence it could serve as a reliable cue for the ovipositing females in a long period of time. We therefore concluded that polarization and not the intensity was the cue guiding females to oviposition sites in the field, while this differentiation was not obvious in the indoor experiment.

\section{Density- and habitat-availability-effects on oviposition behavior}

According to the ideal free distribution (IFD) theory, individuals are $(a)$ assumed to accurately assess habitat quality, $(b)$ are free to move between habitats and as a consequence $(c)$ are distributed according to the habitat quality to maximize the individual fitness (Fretwell and Lucas 1969; see Morris 2006 for review of IFD in fish). Assuming that polarization is a reliable cue for the amount of available resources in the habitat, no eggs were expected to be found in the unpolarized traps according to the IFD theory. Since eggs indeed were deposited in the unpolarized traps in our controlled experiment, isodar 


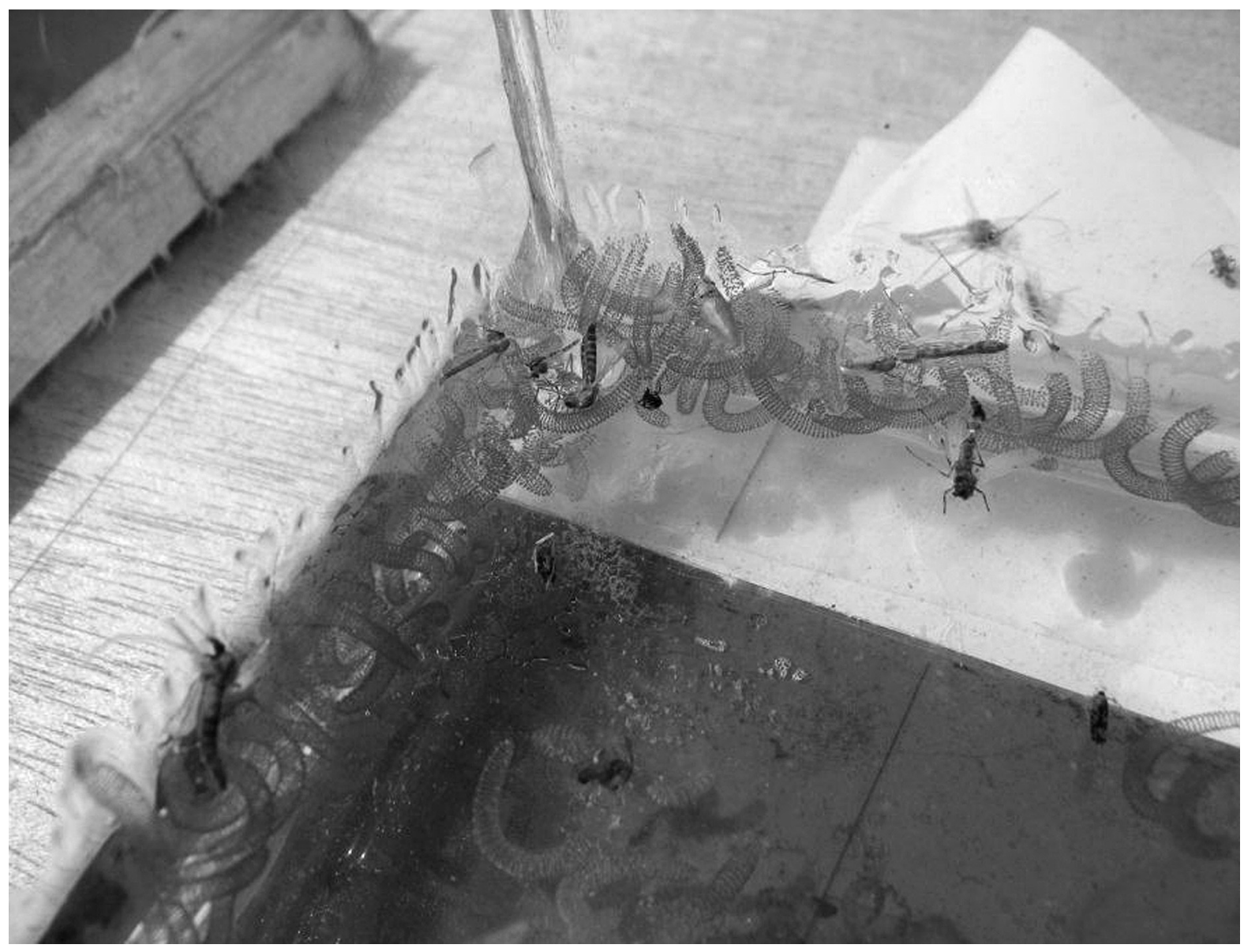

Figure I. Chironomus transvaalensis egg batches (EB, strings) in polarization reflecting oviposition trap. The trap is made of a glass aquarium filled with tap water. The EB are attached to the air-glass-water interface. The dark part is a polarizing sheet placed beneath the aquarium, which fully polarizes the light coming from inside the box (for details see Lerner et al. 2008). Photo: Amit Lerner.

analysis (Morris 1987; 1988) was applied to understand why eggs were found in these habitats (Lerner et al. 2011). In isodar analysis, the number of individuals in the high quality habitat is plotted against the number of individuals in the low quality habitat. The fitted line between them is termed the 'isodar line' and represents a line of equal fitness, which means that an individual will gain equal fitness in both habitats. As individual numbers increase, this analysis represents the decline in the quality of the habitats as a consequence of the increasing individual density. Therefore, this analysis is suitable to explain the presence of individuals in a habitat (EB counts in our study) affected by both the habitat quality and the specimen density. The effect of egg density and habitat availability was examined by comparing the egg counts found in different egg-traps in each of the two experiments. We showed that in the controlled experiment, where oviposition sites were limited, a strong density effect was found as the ratio of sampled egg batches in polarized and unpolarized traps was close to 1 (almost the same amount of eggs in both habitats). In contrast, in the outdoor experiment, the EB ratio found between the polarized and unpolarized sites was in order of magnitude higher, and egg density was an order of magnitude smaller than the density in the indoor experiment ( $\sim .4$ vs. $\sim 4.2 \mathrm{~EB} \mathrm{~cm}^{-1}$ respectively). This can be explained by the effectively unlimited number of oviposition sites available (females could oviposit along the edge of the pond) which has weakened the effects of density and future competition on the offspring.

\section{Visual control of a chironomid population}

The method of luring chironomid females to oviposit in artificial egg traps has been tested on an outdoor chironomid population during March-May 2010. The aim of this experiment was to see if females could be diverted to egg-traps (tubs) by reducing the amount of their high-quality natural oviposition sites (pond edge). The experiment was conducted in the Hayogev waste stabilization pond, Israel $\left(32^{\circ} 36^{\prime} \mathrm{N} 35^{\circ} 10^{\prime} \mathrm{E}\right)$ were a population of Chironomus transvaalensis thrives. A larger pond is located about $400 \mathrm{~m}$ North-West to our test site which could serve as a recruiting source of individuals to the population examined. Ten Styrofoam rafts of $25 \mathrm{~cm} \times 25 \mathrm{~cm}$ were placed in the pond near the shore and the chironomid population was monitored by counting the EB laid on the water-touching edges of the rafts. 
This method has been found to be effective in a previous study (Broza et al. 2003). Ten black tubs filled with 20 liters of pond water were placed on the pond's shore close to the water and parallel to the styrofoam plates. The EBs attached to the plates and in the tubs were counted every morning and were removed right after to clean the tubs and the plates and prepare them for the oviposition cycle of the next evening. After 15 days of sampling, the pond edge which served as the natural oviposition site was covered with a white plastic sheet, $1.5-\mathrm{m}$ wide that reflected unpolarized light, to divert the females to oviposit in the tubs. The pond water surface reflected $\sim 0.7$ partial polarization while the water in the tub reflected 0.4 partial polarization (Figure 2). Samples were taken continuously for 50 days.

During the first 15 sampling days and before the white plastic sheet was placed on the pond's edge, the chironomid population was stable and low with ca. 100-150 EB laid per day both on the plates and in the tubs (Figure 3). Just before placing the white plastic sheet, the chironomid population increased and the EB counts totaled 150 to 300 EB per day. The EB counts in the tubs did not change. However, right after the pond edge was covered in plastic (April 15th, dashed vertical line in Figure 3) and within 10 sampling days, the number of EB in the tubs increased rapidly and were tripled from about 100 to 300 EB per day. This demonstrated that the plastic sheet decreased the availability of the natural habitats, and as a consequence, diverted the females to oviposit in the tubs, increasing the tub's efficiency as an artificial and alternative oviposition site.

\section{Visually-based pest control}

Many animals use visual cues to find appropriate food (e.g. Gerchman et al. 2012) or to find suitable sites to oviposit. In both cases, the preference is fitness related, either directly on the choosing individual or on its offspring. Commercial crops suffer from pests that use fruit color to decide where to lay their eggs. The fruit color is closely related to the stage of ripeness. To synchronize the fruit ripeness with the emergence of the larvae, insects presumably prefer the unripe fruit to lay their eggs. For example, in a laboratory experiment, the papaya fly, Toxotraypana curvicauda, was shown to prefer the unripe green over the ripe yellow papaya fruit for ovipostition (Pena et al. 1986). This interesting finding was not confirmed in the field, nor was it applied to control the fly in a papaya plantation. However, the use of colored sticky plates to catch adult pests is based on the unresolved attraction of the insects to certain colors. Although these color plates have some efficiency, they do not diminish the pest population, and are often combined with chemical or biological methods or just serve to monitor population level in the plantation.

Some studies claim that aquatic insects prefer to oviposit on dark surfaces (e.g. Hoel et al. 2011). However, this experiment was conducted in confined conditions in the lab and lack
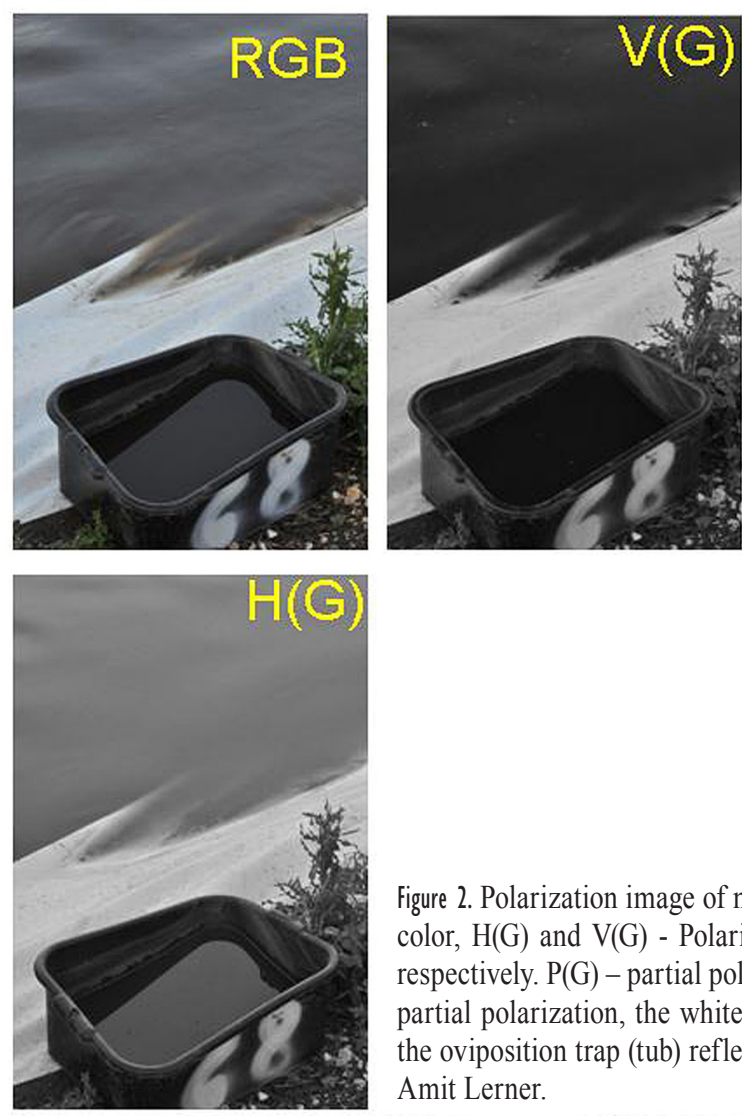
Amit Lerner.
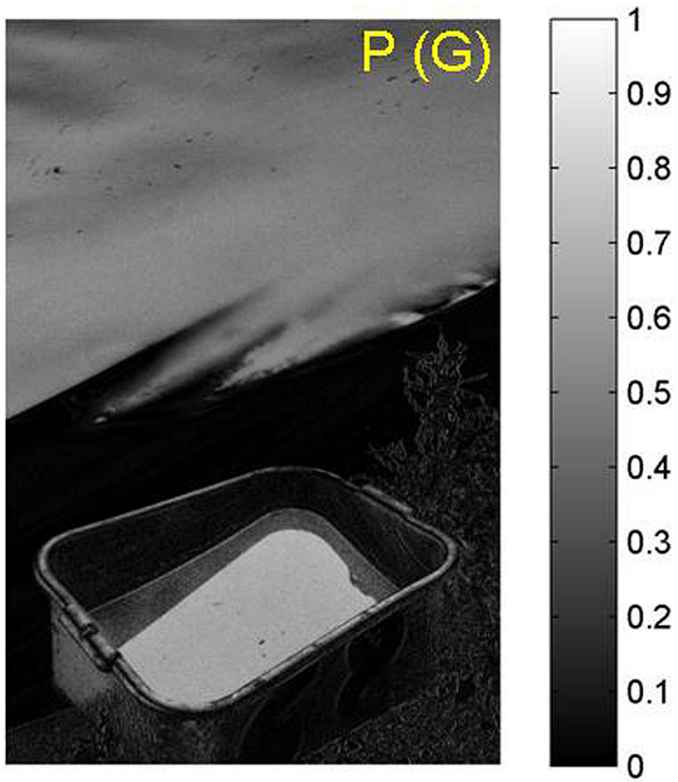

Figure 2. Polarization image of natural and artificial oviposition sites of $C$. transvaalensis. RGB - true color, $\mathrm{H}(\mathrm{G})$ and $\mathrm{V}(\mathrm{G})$ - Polarizer with horizontal and vertical transmission axes in green channel respectively. $\mathrm{P}(\mathrm{G})$ - partial polarization in the green channel. Note that the natural pond reflected 0.7 partial polarization, the white plastic sheet covering the pond edge reflected unpolarized light and the oviposition trap (tub) reflected between 0.4 to 1.0 partial polarization (at Brewster angle). Photo: 


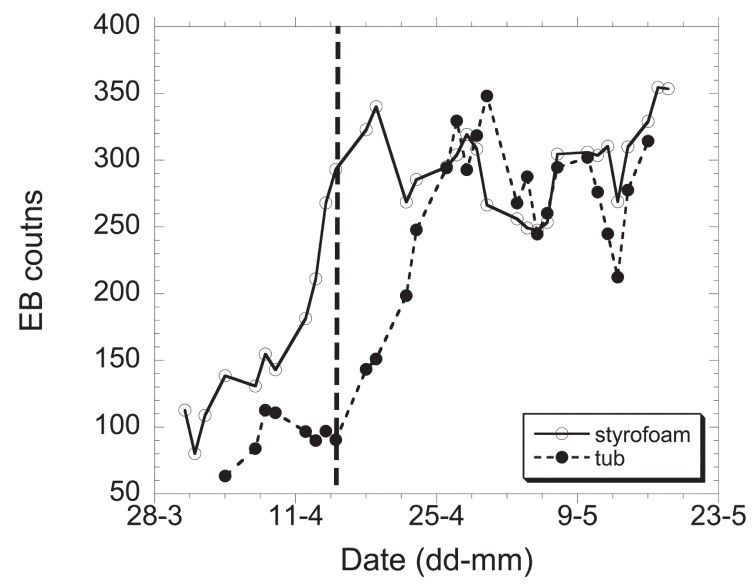

Figure 3. Shift of oviposition to polarized traps after reduction of natural site attractiveness to $C$. transvaalensis. Open circles represent EB counted on white Styrofoam rafts placed in the pond $(n=10)$. Filled circles represent EB counted in black plastic tubs filled with pond water placed on the pond shore $(n=10)$. The dash vertical line represents the day in which the pond edge was covered with white plastic sheet reflecting unpolarized light.

confirmation of the guiding cue in the field. In addition, the effects of brightness (or intensity) and polarization as the guiding cue can easily be confused in cases where these two parameters are not carefully separated and controlled in the experiment. Intensity by itself is known to be positively correlated to insect attraction (positive phototaxis), but the reason is difficult to explain. On the other hand, polarization is negatively dependent on reflectivity (Umow, 1905). Accordingly, polarization reflected from the water surface has been shown to be positively correlated with water turbidity caused by suspended organic carbon in the water, an important food source for larvae of aquatic insects (Lerner et al. 2008). Moreover, turbidity is negatively correlated with the water reflectivity. Therefore, in cases where color is excluded, polarization should be suspected to be the guiding cue for the ovipositing females.

\section{DISCUSSION}

In this review we aim to provide insights on chironomid oviposition behavior and to the possibility to control pest by using traps based on visual cues. This approach can provide an additional solution for controlling infesting insects. It is noneinvasive and fitness related and as such may be highly effective in controlling pests in natural habitats.

In nature, there is no doubt that odor plays an important role in insects site preference and assessment. However, Chironomus transvaalensis provides an important example that ovipositing females may not follow odor but rely on visual cues. This chironomid was found to use polarization and not the intensity reflection as its visual guiding cue to oviposition sites in its natural environment. The use of a stable and reliable visual cue, such as the reflected polarization, provides an easy and quick guide to potentially good sites since polarization is correlated to available larval food. Since misidentification of appropriate oviposition sites is common (e.g. Horváth \& Zeil, 1996; Kriska et al. 2006; Horváth et al., 2009), polarized reflecting egg-traps should be highly effective in controlling species with a similar life-style to that of $C$. transvaalensis.

Of the three characteristics of light, color (or hue), intensity (or brightness) and polarization, the role of intensity in visual attraction is the less explained. It is unclear what benefit intensity provides as a cue, and how it is connected to the fitness of the attracted adult or to its offspring. Color has been found to be a guiding cue (mainly as a good indicator and probably in addition to odor) for certain insect larvae as it indicates the ripeness of the fruit on which the larvae feed. Ovipositing on the fruit in the correct stage of maturity is crucial for survival of the larvae. However, making a trap that is based on the preferred spectrum may not lead to the desired result, and it may not be attractive enough to divert the oviposition of the females. Not more than $50 \%$ efficiency can be expected, as the trap and the fruit will be equally attractive to the female. There might be a part in the spectrum that may be more attractive to the female on which a super-stimulating trap could be constructed, but such favored stimulus has yet to be found. Polarization, on the other hand, may be more effective as it does not appear in its full capacity in nature. Natural surfaces do not reflect more than 0.7 partial polarization (e.g. Horváth et al. 2002) while higher polarization reflections are created by man-made substances such as oil, car paint or window glasses (Kriska et al. 2008; Malik et al. 2008; Horváth et al., 2009). These surfaces can reflect up to 1.0 partial polarization (especially when viewing from Brewster angle or in case they are made of fully polarizing sheets) hence can be more appealing to the insects. Therefore, by building egg traps based on highly polarizing synthetic surfaces, one can expect an increase in the attractiveness to females by up to $30 \%$ compared to natural surfaces, assuming that polarization is the only cue affecting the female choice. For species in which polarization is closely correlated to their fitness, polarized traps should be a highly effective tool for pest control.

Another important lesson that our studies on chironomid oviposition taught us, was that the oviposition behavior in the lab did not resemble the one in nature. In the lab, we provided a limited number of oviposition sites in a multiple-choice experiment, and as a response, the females concentrated their oviposition in space and time. This was not the case in the field as there were many suitable oviposition sites, and in practice these exceeded the number needed by the insect population. Thus in nature, not all sites (e.g. fruits) will be infected, and the egg density per site may be very low (less than one). This means that it is difficult to control the pest population and that the number of traps needed to do this might be very high. It also requires traps that are much more effective than traps used in the lab, for instance by strengthening of the visual cue. 


\section{ACKNOWLEDGMENTS}

We would like to thank to Ran Peretz for counting EBs in the control experiment, and to Emek Israel Regional Council and its head engineer Noam Graif for allowing us to use the Hayogev waste stabilization station. This research was partly supported by a Council of Higher Education Post-Doctoral fellowship to AL, by an Israeli Science Foundation Grant \#081/10 to NS, and by the "Applied Sciences Funds of The Hebrew University, Israel" Grant to $\mathrm{CH}$.

\section{REFERENCES}

Armitage PD, Cranston PS, Pinder LCV. 1995. The Chironomidae: the biology and ecology of non-biting midges. Chapman \& Hall. $572 \mathrm{p}$.

Bernáth B, Horváth G, Gál J, Fekete G, Meyer-Rochow VB. 2008. Polarized light and oviposition site selection in the yellow fever mosquito: No evidence for positive polarotaxis in Aedes aegypti. Vision Research 48(13): 1449-1455.

Broza M, Halpern M. 2001. Pathogen reservoirs - Chironomid egg masses and Vibrio cholerae. Nature 412(6842): 40.

Broza M, Halpern M, Teltsch B, Porat R, Gasith A. 1998. Shock chloramination: Potential treatment for chironomidae (Diptera) larvae nuisance abatement in water supply systems. Journal of Economic Entomology 91(4): 834-840.

Broza M, Halpern M, Gahanma L, Inbar M. 2003. Nuisance chironomids in waste water stabilization ponds: monitoring and action threshold assessment based on public complaints. Journal of Vector Ecology 28(1): 31-36.

Broza M, Gancz H, Kashi Y. 2008. The association between nonbiting midges and Vibrio cholerae. Environmental Microbiology 10(12): 3193-3200.

Danthanarayana W, Dashper S. 1986. Response of some night flying insects to polarized light. In: W. D (ed.). Insect flight: dispersal and migration. Springer. pp 120-127.

Fretwell SD, Lucas HL. 1969. On territorial behavior and other factors influencing habitat distribution in birds. I. Theoretical development. Acta Biotheoretica 19: 16-36.

Gerchman Y, Dodek I, Petichov R, Yerushalmi Y, Lerner A, Keasar T. 2012. Beyond pollinator attraction: extra-floral displays deter herbivores in a Mediterranean annual plant. Evolutionary Ecology 26: 499-512. 10.1007/s10682-011-9509-3

Halpern M, Broza YB, Mittler S, Arakawa E, Broza M. 2004. Chironomid egg masses as a natural reservoir of Vibrio cholerae non-O1 and non-O139 in freshwater habitats. Microbial Ecology 47(4): 341-349.

Hoel DF, Obenauer PJ, Clark M, Smith R, Hughes TH, Larson RT, Diclaros JW, Allan SA. 2011. Efficacy of ovitrap colors and patterns for attracting Aedes albopictus at suburban field sites in north-central Florida. Journal of the American Mosquito Control Association 27(3): 245-251.

Horváth G, Zeil J. 1996. Kuwait oil lakes as insect traps. Nature 379(6563): 303-304.

Horváth G, Varjú D. 2004. Polarized light in animal vision: polarization patterns in nature. Springer Verlag. 470p.

Horváth G, Gal J, Labhart T, Wehner R. 2002. Does reflection polarization by plants influence colour perception in insects? Polarimetric measurements applied to a polarization-sensitive model retina of Papilio butterflies. Journal of Experimental Biology 205(21): 3281-3298.

Horváth G, Kriska G, Malik P, Robertson B. 2009. Polarized light pollution: a new kind of ecological photopollution. Frontiers in Ecology and the Environment 7(6): 317-325.

Horváth G, Móra A, Bernáth B, Kriska G. 2011. Polarotaxis in nonbiting midges: Female chironomids are attracted to horizontally polarized light. Physiology \& Behavior 104(5): 1010-1015.

Kokkinn MJ, Williams WD. 1989. An experimental study of phototactic response of Tanytarsus barbitarsis Freeman (Diptera: Chironomidae). Australiean Journal of Marine Freshwater Research 40: 693-702.

Kriska G, Csabai Z, Boda P, Malik P, Horváth G. 2006. Why do red and dark-coloured cars lure aquatic insects? The attraction of water insects to car paintwork explained by reflectionpolarization signals. Proceedings of the Royal Society BBiological Sciences 273(1594): 1667-1671.

Kriska G, Malik P, Szivak I, Horváth G. 2008. Glass buildings on river banks as "polarized light traps" for mass-swarming polarotactic caddis flies. Naturwissenschaften 95(5): 461-467.

Lerner A, Meltser N, Sapir N, Erlick C, Shashar N, Broza M. 2008. Reflected polarization guides chironomid females to oviposition sites. Journal of Experimental Biology 211(22): 3536-3543.

Lerner A, Sapir N, Erlick C, Meltser N, Broza M, Shashar N. 2011. Habitat availability mediates chironomid density dependent oviposition. Oecologia 165(4): 905-914.

Malik P, Hegedus R, Kriska G, Horváth G. 2008. Imaging polarimetry of glass buildings: why do vertical glass surfaces attract polarotactic insects? Applied Optics 47(24): 4361-4374.

Meltser N, Kashi Y, Broza M. 2008. Does polarized light guide chironomids to navigate toward water surfaces? Boletin do Museu Municipal do Funchal (História Natural) 13: 141-149.

Morris DW. 1987. Tests of Density-Dependent Habitat Selection in a Patchy Environment. Ecological Monographs 57(4): 270-281.

Morris DW. 1988. Habitat-dependent population regulation and community structure. Evolutionary Ecology 2(3): 253-269.

Morris DW. 2006. Ecology - moving to the ideal free home. Nature 443(7112): 645-646.

Paz S, Broza M. 2007. Wind direction and its linkage with Vibrio cholerae dissemination. Environmental Health Perspectives 115(2): 195-200.

Pena JE, Baranowski RM, Litz RE. 1986. Oviposition of the papaya fruit fly Toxotraypana curvicauda Gerstaecker as affected by fruit maturity. Florida Entomologist 69(2): 344-348.

Schwind R. 1991. Polarization vision in water insects and insects living on a moist substrate. Journal of Comparative Physiology a-Sensory Neural and Behavioral Physiology 169(5): 531-540.

Umow N. 1905. Chromatische depolarisation durchlichtzerstreuung. Physikalische Zeitschrift 6: 674-676. 